Licença CC BY: Artigo distribuído sob os termos Creative Commons, permite uso e distribuição irrestrita em qualquer meio desde que $o$ autor credite a fonte original.

\section{ACESSIBILIDADE EM MEIOS DE HOSPEDAGEM: O USO DE PROCESSOS INOVADORES NO ATENDIMENTO DAS NECESSIDADES DO TURISTA COM DEFICIÊNCIA}

\author{
ACCESSIBILITY IN ACCOMMODATION ESTABLISHMENTS: THE \\ USE OF INNOVATIVE PROCESSES TO MEET THE NEEDS OF \\ TOURISTS WITH DISABILITIES
}

\author{
MARKLEA DA CUNHA FERST ${ }^{1}$ \\ JULIA ISMAR SILVA DE SOUZA ${ }^{1}$ \\ HELEN RITA MENEZES COUTINHO'
}

\section{'UNIVERSIDADE DO ESTADO DO AMAZONAS, MANAUS, AMAZONAS, BRASIL}

\begin{abstract}
RESUMO: O objetivo geral do artigo é analisar a utilização de processos inovadores para o atendimento das necessidades da pessoa com deficiência nos meios de hospedagem localizados próximos aos atrativos do Centro Histórico de Manaus. Esta pesquisa tem como objetivos específicos: caracterizar a deficiência e o turismo acessível a partir da literatura acadêmica e da legislação brasileira; identificar na legislação do Brasil quais os requisitos devem ser observados pela rede hoteleira para o atendimento das necessidades da pessoa com deficiência; caracterizar a inovação na gestão hoteleira a partir da literatura acadêmica; analisar se existem práticas inovadoras de gestão hoteleira para o atendimento das necessidades da pessoa com deficiência pelos meios de hospedagem. Como procedimentos metodológicos, utilizou-se o método indutivo, com abordagem quantitativa, com objetivos de pesquisa exploratória e descritiva e com procedimentos bibliográficos e de campo, com uma amostra de 18 meios de hospedagem. A pesquisa constatou que nos meios de hospedagem localizados próximos aos atrativos do Centro Histórico de Manaus não são utilizados processos inovadores na gestão de atendimento do segmento de clientes com deficiência. O diferencial deste artigo é a abordagem específica dos requisitos previstos na legislação a serem atendidos pelos meios de hospedagem, ante o recente Decreto regulamentador promulgado em 2018.
\end{abstract}

Marklea Professora Assistente da Universidade do Estado do Amazonas, Manaus, Amazonas, Brasil. Doutoranda em Turismo e Hotelaria na Universidade do Vale do Itajaí, Balneário Camboriú, Santa Catarina, Brasil. E-mail: mferst@uea.edu.br. Orcid: 0000-0003-4399-8870.

Julia Professora da Universidade do Estado do Amazonas, Manaus, Amazonas, Brasil. Doutoranda em Turismo e Hotelaria na Universidade do Vale do Itajaí, Balneário Camboriú, Santa Catarina, Brasil. E-mail: jisds.tur $16 @$ vea.edu.br. Orcid: 0000-0002-9042-4418

Helen Professora da Universidade do Estado do Amazonas, Manaus, Amazonas, Brasil. Doutoranda em Turismo e Hotelaria na Universidade do Vale do Itajaí, Balneário Camboriú, Santa Catarina, Brasil. E-mail: hcoutinho@vea.edu.br. Orcid: 0000-0001-7205-5914 
PALAVRAS-CHAVE: Turismo acessível. Meios de hospedagem. Inovação.

\begin{abstract}
The general objective of the article is to analyze the use of innovative processes to meet the needs of people with disabilities in accommodation establishments located near the attractions of the Historic Center of Manaus. Its specific objectives are: to characterize disability and accessible tourism, based on the academic literature and the Brazilian legislation; to identify, in the Brazilian legislation, the requirements that must be met by the hotel network in order to meet the needs of people with disabilities; to characterize innovation in hotel management based on the academic literature and; to analyze whether there are innovative hotel management practices for meeting the needs of people with disabilities through accommodation. In terms of methodological procedures, the inductive method was used, with the quantitative approach, exploratory and descriptive research objectives, and bibliographic and field procedures. The sample included eighteen accommodation establishments. The research found that the accommodation establishments located near the attractions of the Historic Center of Manaus do not use innovative processes for the care management of guests with disabilities. The unique point of this article is that it addresses the requirements for accommodation establishments set out in the legislation, in light of the recent regulatory Decree issued in 2018.
\end{abstract}

KEYWORDS: Accessible tourism. Accommodation establishments. Innovation.

RESUMEN: El objetivo general del artículo es analizar el uso de procesos innovadores para satisfacer las necesidades de la persona con discapacidad en los medios de alojamiento situados cerca de los atractivos del Centro Histórico de Manaus. Esta investigación tiene como objetivos específicos: caracterizar la discapacidad y el turismo accesible a partir de la literatura académica y legislación brasileña; identificar en la legislación de Brasil cuáles requisitos deben ser observados por la red hotelera para atender las necesidades de la persona con discapacidad; caracterizar la innovación en la gestión hotelera a partir de la literatura académica; analizar si existen prácticas innovadoras de gestión hotelera para atender las necesidades de la persona con discapacidad por los medios de alojamiento. Como procedimientos metodológicos, se utilizó el método inductivo; con enfoque cuantitativo; con objetivos de investigación exploratoria y descriptiva; y con procedimientos bibliográficos y de campo; con una muestra de 18 medios de hospedaje. La investigación encontró que en los medios de alojamiento situados cerca de los atractivos del Centro Histórico de Manaus no se utiliza procesos innovadores en la gestión de la atención del segmento de clientes con discapacidad. El diferencial de este artículo es la demostración específica de los requisitos previstos en la legislación a ser atendidos por los medios de alojamiento, ante el reciente Decreto regulador promulgado en 2018.

PALABRAS CLAVE: Turismo accesible. Medios de alojamiento. Innovación.

\title{
INTRODUÇÃO
}

O turismo vem contribuindo substancialmente para o desenvolvimento da economia mundial e se desenvolvido de forma crescente (Oliveira, Franzen, \& Varella, 2016). Estimativas do Conselho Mundial de Viagens e Turismo (WTTC) (BRASIL, 2018b) indicam ter sido o turismo responsável pela criação de 7 milhões de empregos no mundo no ano de 2017, ou seja, o equivalente a 10,4\% do Produto Interno Bruto (PIB) global, tendo um crescimento estimado de 3,8\% para a próxima década. Incontestável, assim, seu importante papel na economia. 
Certamente que as estimativas promissoras na área turística refletem invariavelmente no setor hoteleiro, uma vez que 49,6\% dos turistas hospedam-se em hotéis, flats, resorts ou pousadas (BRASIL, 2017). Vale destacar, ainda, que a hotelaria é o setor que mais gera empregos no turismo (Meira \& Anjos, 2013).

De outro lado, o turismo acessível tem sido considerado por diversos autores como um potencial e rentável mercado, pois além do grande número de pessoas com deficiência no mundo - mais de um bilhão de pessoas, de acordo com a Organização Mundial da Saúde (World Health Organization, 2012) -, tem-se ainda o constante crescimento da população idosa, e o fato de que a pessoa com deficiência costuma viajar acompanhada, ou seja, este número de potenciais turistas é bastante expressivo (Card, Cole \& Humphrey, 2006, Huh \& Singh, 2007, Dávid \& Kiss, 2011, Babaita, 2012, Rabontu, 2018).

Outro fator relevante a ser observado é a questão da hospitalidade, e, sendo o acolhimento uma das principais características da hospitalidade, um bom acolhimento irá satisfazer o turista e culminar em uma divulgação positiva do local visitado (Camara \& Fonseca Filho, 2019).

Sendo assim, considerando o público de pessoas com deficiência e idosos, bem como o fato de que as necessidades de ambos são semelhantes, aliado ao crescimento estimado do turismo, da importância da hospitalidade e, consequentemente, dos meios de hospedagem, analisar a acessibilidade destes vai além da averiguação do cumprimento da legislação vigente relativa à infraestrutura que deve ser observada pelos meios de hospedagens, sendo importante uma análise mais robusta, a fim de dar subsídios aos operadores dos setores voltados ao turismo para o investimento em infraestrutura para atender às necessidades do público-alvo do turismo acessível.

Entretanto, em que pese a potencialidade do turismo acessível, o setor turístico pouco tem se preocupado com as preferências de instalação das pessoas com deficiência ou mobilidade reduzida (Lyu, 2017), sendo necessária a adoção de processos inovadores para atender da melhor forma possível a todos os perfis de turistas e suas necessidades particulares.

Quando se fala em processos inovadores, entenda-se não apenas o atendimento básico das normas previstas na legislação, tais como rampas de acesso com as medidas de inclinação indicadas na lei, mas ir além, criar facilidades que importem em maior conforto à pessoa com deficiência ou mobilidade reduzida, propiciando-lhe uma melhor experiência turística, tais como a utilização das chamadas "cadeiras anfíbias" para que cadeirantes possam utilizar a piscina do hotel ou a praia nos resorts.

Desta feita, este trabalho visa analisar se meios de hospedagem localizados próximos aos atrativos do Centro Histórico de Manaus atendem de maneira inovadora as necessidades da pessoa com deficiência ou mobilidade reduzida, tendo como objetivo geral analisar a utilização de processos inovadores para o atendimento das necessidades da pessoa com deficiência ou mobilidade reduzida nos meios de hospedagem localizados próximos aos atrativos do Centro Histórico de Manaus. 
E para atingir o objetivo geral, elaboraram-se os seguintes objetivos específicos: caracterizar a deficiência e o turismo acessivel a partir da literatura acadêmica e legislação brasileira; identificar na legislação do Brasil quais os requisitos devem ser observados pela rede hoteleira para o atendimento das necessidades da pessoa com deficiência ou mobilidade reduzida; caracterizar a inovação na gestão hoteleira a partir da literatura acadêmica; analisar se existem práticas inovadoras de gestão hoteleira para o atendimento das necessidades da pessoa com deficiência ou mobilidade reduzida pelos meios de hospedagem próximos aos atrativos do Centro Histórico de Manaus.

No desenvolvimento da pesquisa, além da revisão bibliográfica para subsidiar as definições sobre o turismo acessível e seus agentes, os requisitos de acessibilidade previstos na legislação a serem atendidos pelos meios de hospedagem e a importância da inovação para o processo de desempenho das organizações de hospedagem, foi realizada uma pesquisa de campo para averiguar se os meios de hospedagem estão utilizando processos inovadores no atendimento das necessidades da pessoa com deficiência ou mobilidade reduzida, e de que forma, demonstrando-se, ao final, como um meio de hospedagem acessível pode contribuir para se alcançar um maior desempenho geral da empresa hoteleira.

Entende-se que a pesquisa apresentada irá contribuir futuramente à sensibilização de profissionais da área do turismo, especialmente gestores da área de hotelaria da importância de se promover processos inovadores para o atendimento das normas de acessibilidade, o que deve culminar no desenvolvimento de ações eficazes para $o$ atendimento das necessidades das pessoas com deficiência ou mobilidade reduzida em meios de hospedagem e o desenvolvimento do turismo acessível.

\section{DIREITOS DA PESSOA COM DEFICIÊNCIA E TURISMO ACESSÍVEL}

A acessibilidade nos meios de hospedagem é fator determinante para a inclusão da pessoa com deficiência ou mobilidade reduzida no turismo, devendo os meios de hospedagem estar devidamente adaptados para receber este público, tanto no que diz respeito à qualificação de seus funcionários quanto na sua infraestrutura, posto ser a ausência de acessibilidade um impeditivo para que a pessoa com deficiência pratique o turismo (Duarte, Borda, Moura \& Spexia, 2015, Michopoulou, Darcy, Ambrose \& Buhalis, 2015).

Nos termos do artigo $2^{\circ}$ da Lei 13.146, de 06 de julho de 2015 (Brasil, 2015), considera-se pessoa com deficiência aquela que tem impedimento de longo prazo de natureza física, mental, intelectual ou sensorial, o qual, em interação com uma ou mais barreiras, pode obstruir sua participação plena e efetiva na sociedade em igualdade de condições com as demais pessoas, assegurando, ainda, em seu artigo 42, o direito à cultura, ao esporte, ao turismo e ao lazer em igualdade de oportunidades com as demais pessoas.

Importante destacar que a Lei 13.146/2015 aplica-se também à pessoa com mobilidade reduzida, que é definida no inciso IX, do artigo $3^{\circ}$ como "aquela que tenha, 
por qualquer motivo, dificuldade de movimentação, permanente ou temporária, gerando redução efetiva da mobilidade, da flexibilidade, da coordenação motora ou da percepção", deixando expressamente previsto que se incluem no conceito os idosos, as gestantes, as lactantes, a pessoa com criança de colo e o obeso (Brasil, 2015).

Do conceito legal previsto na legislação vigente se percebe com clareza ser o turismo um direito da pessoa com deficiência ou mobilidade reduzida. Vale ressaltar que tal direito previsto na legislação brasileira decorre da Convenção da ONU dos direitos da pessoa com deficiência e seu protocolo especial, ou seja, tem-se um direito universal ao turismo e ao lazer garantido às pessoas com deficiência, surgindo, assim, o segmento denominado turismo acessível (Biddulph \& Scheyvens, 2018).

Quando se fala em turismo acessível, entende-se como um conjunto de instalações e práticas para permitir ou melhorar a experiência turística para um amplo espectro de pessoas (Cole \& Morgan, 2010).

O atendimento de elementos como visão, audição, mobilidade e dimensões cognitivas do acesso, que devem funcionar de maneira autônoma e com igualdade, por intermédio da prestação de serviços, ambientes e produtos turísticos devidamente projetados para este fim, também devem ser considerados na definição do que se considera como turismo acessível (Offei, Acheampong, Appiah-Brempong, Okyere \& Owusu, 2017; Buhalis \& Sinarta, 2019).

Ainda que se trate de um novo conceito, o turismo para pessoas com deficiência é um expressivo mercado mundial em franco desenvolvimento (Jaremen, 2014, Allan, 2015, Williams et al., 2017, Rabontu, 2018), merecendo a atenção do setor turístico e dos estudiosos do turismo.

\section{NORMAS BRASILEIRAS DE DESIGN UNIVERSAL PARA OS MEIOS DE HOSPEDAGEM}

O turismo acessível implica na utilização do modelo de design universal, a fim de permitir que qualquer pessoa utilize - sem dificuldades - diversos produtos e ambientes (Buhalis \& Darcy, 2011, Michopoulou et al., 2015).

O conceito de desenho universal nos termos da legislação brasileira é definido como a concepção de produtos, ambientes, programas e serviços a serem usados por todas as pessoas, sem necessidade de adaptação ou de projeto específico, incluindo os recursos de tecnologia assistiva (Brasil, 2015), prevendo o artigo 45 do Estatuto da Pessoa com Deficiência (Brasil, 2015) que hotéis, pousadas e similares devem ser construídos observando-se os princípios do desenho universal, além de adotar todos os meios de acessibilidade nos termos da legislação em vigor. 
A acessibilidade é caracterizada, segundo a Lei 13.146/2015, como a possibilidade e a condição de alcance de espaços, mobiliários, equipamentos urbanos, edificações, transporte, informação e comunicação, bem como serviços e instalações abertos ao público de uso público ou privados, de uso coletivo, tanto na zona urbana quanto na zona rural, para a utilização com autonomia e segurança por parte da pessoa com deficiência ou mobilidade reduzida (Brasil, 2015).

Conforme previsto no artigo 23 da Política Nacional do Turismo (BRASIL, 2008), os meios de hospedagem são considerados os empreendimentos ou os estabelecimentos, independentemente de sua constituição, destinados a prestar serviços de alojamento temporário, ofertados em unidades de frequência individual e de uso exclusivo do hóspede, bem como outros serviços necessários aos usuários, mediante adoção de instrumento contratual, tácito ou expresso, e cobrança diária.

De acordo com o previsto no Decreto n 9.296 , de $1^{\circ}$ de março de 2018 (BRASIL, 2018a), a concepção e a implantação de projetos arquitetônicos de hotéis, pousadas e estruturas similares deverão atender aos princípios do desenho universal e ter como referências básicas as normas técnicas de acessibilidade da Associação Brasileira de Normas Técnicas (ABNT), e a legislação específica.

Nos termos do referido decreto, os meios de hospedagem deverão disponibilizar, no mínimo, cinco por cento dos dormitóriosa, respeitado o mínimo de um, com as características construtivas e os recursos de acessibilidade previstos no Quadro 1. 


\section{Quadro 1: Características construtivas e recursos de acessibilidade para 5\% dos quartos}

\begin{tabular}{|c|c|}
\hline Item & Características construtivas \\
\hline 01 & $\begin{array}{l}\text { Dimensões de acesso, de circulação, de manobra, de alcance e de mobiliário estabelecidas na norma téc- } \\
\text { nica de acessibilidade da Associação Brasileira de Normas Técnicas (ABNT) para dormitórios acessíveis. }\end{array}$ \\
\hline 02 & $\begin{array}{l}\text { Banheiro que atenda integralmente as especificações estabelecidas na norma técnica de acessibilidade } \\
\text { da ABNT. }\end{array}$ \\
\hline 03 & $\begin{array}{l}\text { Chuveiro equipado com barra deslizante, desviador para ducha manual e controle de fluxo (ducha/chu- } \\
\text { veiro) na ducha manual (chuveirinho), o qual deverá estar sempre posicionado na altura mais baixa } \\
\text { quando da chegada do hóspede. }\end{array}$ \\
\hline 04 & $\begin{array}{l}\text { Condições de circulação, aproximação e alcance de utensílios e instalações estabelecidas na norma téc- } \\
\text { nica de acessibilidade da ABNT, quando houver cozinha ou similar na unidade. }\end{array}$ \\
\hline 05 & Olhos-mágicos instalados nas portas nas alturas de cento e vinte e cento e sessenta centímetros. \\
\hline 06 & $\begin{array}{l}\text { Sistema magnético de tranca das portas dos dormitórios que permita autonomia ao hóspede com defi- } \\
\text { ciência visual, surdo ou surdo-cego, além de informações em relevo, ranhuras ou cortes nos escaninhos } \\
\text { de leitura e nos cartões magnéticos. }\end{array}$ \\
\hline 07 & Campainha (batidas na porta) sonora e luminosa intermitente (flash) na cor amarela. \\
\hline 08 & $\begin{array}{l}\text { Sinalização de emergência para os casos de incêndio ou perigo, sonora e luminosa intermitente (flash) na } \\
\text { cor vermelha. }\end{array}$ \\
\hline 09 & Aparelho de televisão com dispositivos receptores de legenda oculta e de áudio secundário. \\
\hline 10 & Telefone com tipologia ampliada e com amplificador de sinal. \\
\hline
\end{tabular}

Fonte: Anexo I do Decreto no 9.296/2018.

O hotel deve, ainda, disponibilizar, para $95 \%^{\mathrm{b}}$ dos demais dormitórios, os recursos de acessibilidades descritos no Quadro 2. 


\section{Quadro 2: Recursos de acessibilidade para 95\% dos quartos}

\begin{tabular}{|c|c|}
\hline Item & Recursos de Acessibilidade \\
\hline 01 & $\begin{array}{l}\text { Vão de passagem livre, mínimo de } 80 \text { centímetros para a porta da unidade habitacional e para a porta } \\
\text { do banheiro. }\end{array}$ \\
\hline 02 & Barra de apoio no box do chuveiro. \\
\hline 03 & $\begin{array}{l}\text { Chuveiro equipado com barra deslizante, desviador para ducha manual e controle de fluxo na ducha ma- } \\
\text { nual, o qual deverá estar sempre posicionado na altura mais baixa, na ocasião de chegada do hóspede. }\end{array}$ \\
\hline 04 & Olhos-mágicos instalados nas portas nas alturas de cento e vinte e cento e sessenta centímetros. \\
\hline 05 & Campainha sonora e luminosa intermitente na cor vermelha. \\
\hline 06 & $\begin{array}{l}\text { Sistema magnético de tranca das portas dos dormitórios, que permita autonomia ao hóspede com defi- } \\
\text { ciência visual, surdo ou surdo-cego, além de informações em relevo, ranhuras ou cortes nos escaninhos } \\
\text { de leitura e nos cartões magnéticos. }\end{array}$ \\
\hline 07 & $\begin{array}{l}\text { Sinalização de emergência, para os casos de incêndio ou perigo, sonora e luminosa intermitente, na cor } \\
\text { vermelha. }\end{array}$ \\
\hline 08 & $\begin{array}{l}\text { Aparelho de televisão com dispositivos receptores de legenda oculta e de áudio secundário, quando a } \\
\text { unidade UH disponibilizar esse tipo de aparelho. }\end{array}$ \\
\hline 09 & $\begin{array}{l}\text { Telefone com tipologia ampliada e com amplificador de sinal, quando a UH disponibilizar esse tipo de } \\
\text { aparelho }\end{array}$ \\
\hline
\end{tabular}

Fonte: Anexo II do Decreto no 9.296/2018.

Por fim, a rede de meios de hospedagem deve disponibilizar, quando solicitado pelo hóspede, os recursos dispostos no Quadro 3. O hóspede deve fazer a solicitação no momento da reserva, não o fazendo, o estabelecimento tem o prazo de 24 horas para atender ao pedido, a contar da data do requerimento pelo hóspede (BRASIL, 2018a).

\section{Quadro 3: Ajudas técnicas e recursos de acessibilidade exigíveis sob demanda}

\begin{tabular}{|c|c|}
\hline Item & Recursos de Acessibilidade \\
\hline 01 & Cadeiras de roda. \\
\hline 02 & Cadeiras adaptadas para banho. \\
\hline 03 & Materiais de higiene identificados em braille e embalagens em formatos diferentes. \\
\hline 04 & $\begin{array}{l}\text { Materiais impressos disponíveis em formato digital, braille, fonte ampliada com contraste, a exemplo } \\
\text { de formulários impressos, informações sobre facilidades e serviços oferecidos, dentre outros, feitos sob } \\
\text { demanda. }\end{array}$ \\
\hline 05 & Cardápio em braille e fonte ampliada com contraste. \\
\hline 06 & Relógio despertador/alarme vibratório \\
\hline 07 & $\begin{array}{l}\text { Dispositivos móveis com chamada em vídeo e mensagem disponibilizados nas áreas comuns do estabe- } \\
\text { lecimento ou aplicativo de comunicação criado nos termos estabelecidos no Título IV da Resolução no } \\
667 \text {, de } 30 \text { de maio de } 2016 \text {, da Anatel, que aprova o Regulamento Geral de Acessibilidade em Serviços } \\
\text { de Telecomunicações de interesse coletivo. }\end{array}$ \\
\hline
\end{tabular}

Fonte: Anexo III do Decreto no 9.296/2018. 
Os princípios do desenho universal previstos na legislação buscam assegurar que o estabelecimento possa receber o maior número de hóspedes, independentemente de sua condição física, mental, intelectual ou sensorial, garantindo a todos, sem distinção, que possam desfrutar de todas as comodidades disponibilizadas pelo meio de hospedagem, devendo todas as áreas de livre acesso aos hóspedes observar as normas da ABNT e as normas aplicáveis às edificações de uso coletivo previstas no Decreto $n^{\circ}$ 5.296, de 2 de dezembro de 2004. (BRASIL, 2018a).

É importante a padronização dos produtos e dos serviços tradicionais, devendo ao setor turístico "usar padrões de interface que correspondam aos das ajudas técnicas", propiciando, assim, a plena utilização pelas pessoas com deficiência (Michopoulou et al., 2015).

\section{INOVAÇÃO E GESTÃO HOTELEIRA}

O principal indicador de desempenho de empresas de prestação de serviços é o gerenciamento bem-sucedido do processo de produção de serviços. Ou seja, a capacidade gerencial para criar, implementar e gerenciar processos, desenvolvendo, assim, maior valor para o cliente, é o que distingue as empresas de sucesso (TweneboahKoduah, Anning-Dorson \& Nyamekye, 2018).

Estudos indicam que quanto mais acentuada a inovação, melhor o desempenho da empresa (Hertog, Gallouj \& Segers, 2011, Sipe, 2018). TweneboahKoduah e Colab (2018) defendem que tanto a inovação quanto a personalização, combinadas ou não, são elementos essenciais na melhoria do desempenho financeiro e não financeiro das empresas que atuam em meios de hospedagem, destacando, contudo, que quando combinadas - inovação de processos e personalização -, temse níveis mais altos de desempenho nas empresas, uma vez que possuem propriedades que se complementam.

O desenvolvimento da economia e a livre concorrência exigem que as organizações passem de um mero serviço para uma "economia de experiência", sendo essencial que reinventem seus "scorecards de desempenho de negócios para refletir práticas que colocam a experiência de co-criação de hóspedes no centro de seus esforços de inovação" (Sipe, 2018, p. 1).

Assim sendo, conforme destacam Meira e Anjos (2013, p. 2), "a busca pela excelência através da utilização adequada dos recursos, a fim de alcançar maiores índices de lucratividade, não é mais considerada como diferencial e sim uma estratégia de sobrevivência no mercado".

Desta feita, considerando que para se alcançar um desempenho geral elevado nas empresas de meios de hospedagem é preciso que a capacidade de personalização tenha como objetivo melhorar a capacidade de inovação (Tweneboah-Koduah et al., 2018), e considerando as tendências do turismo acessível, pretende-se neste estudo verificar a existência de processos inovadores nos meios de hospedagem. Optou-se, por conveniência, analisar os hotéis próximos ao centro histórico de Manaus, podendo este estudo ser replicado em outros estabelecimentos e localidades. 


\section{PROCEDIMENTOS METODOLÓGICOS}

Trata-se de um trabalho que utilizou o método indutivo. Percebe-se como indutivo na medida em que se buscaram, a partir da análise dos dados obtidos por meio dos respondentes, a existência e a utilização de procedimentos inovadores para atender as necessidades da pessoa com deficiência ou mobilidade reduzida. $O$ presente estudo se classifica, ainda, relativo à finalidade, como básica. Já no tocante à forma de caracterização, trata-se de pesquisa quantitativa (Sampieri, Collado \& Lucio, 2015) e, quanto aos fins, como exploratória e descritiva, uma vez que os eventos foram observados, registrados, analisados, classificados e interpretados.

Por fim, quanto aos procedimentos, para esta pesquisa, foi feito um levantamento bibliográfico e trabalhos de campo (Cooper \& Schindler, 2016) em virtude da utilização de técnicas padronizadas de coleta de dados que permitiram a análise do perfil de atendimento às normas de acessibilidade dos meios de hospedagem nas proximidades do centro histórico de Manaus, onde os dados foram analisados com a utilização de recursos estatísticos, e avaliar a utilização de procedimentos inovadores para o atendimento das necessidades deste público.

Para alcançar o objetivo geral deste artigo, foi realizada uma pesquisa empírica numa amostra não probabilística por conveniência (Cooper \& Schindler, 2016), com 18 meios de hospedagem. Teve como respondentes 8 gestores e 10 funcionários com cargos operacionais.

Os dados foram coletados nos meses de março e abril de 2019, utilizando-se o instrumento de coleta de dados survey monkey. Para a aplicação do questionário, foram realizadas visitas aos hotéis e solicitado que os gestores respondessem ao questionário. Na impossibilidade dos gestores, outros funcionários preencheram o questionário. Para fins de análise neste trabalho, apenas se levaram em consideração as respostas dos funcionários operacionais relativas ao conhecimento sobre a legislação a respeito dos direitos da pessoa com deficiência e no tocante a processos inovadores para o atendimento das necessidades deste segmento de clientes, nas demais questões, levou-se em consideração apenas os respondentes gestores.

O formulário de coleta de dados foi um questionário de 21 perguntas fechadas e 01 pergunta aberta, formuladas a partir dos estudos anteriores das autoras relativos à acessibilidade (Buhalis \& Sinarta, 2019, Var, Yesiltas, Yayli \& Öztürk, 2011), à influência da acessibilidade para a escolha do local turístico (Michopoulou et al., 2015, Smith, Amorim, \& Soares, 2013), às atitudes de gerentes em relação às pessoas com deficiência na rede hoteleira (Paez \& Arendt, 2014) e à avaliação de hotéis para acomodar turistas com deficiência (Tantawy, Kim, \& Pyo, 2005). A análise de dados foi realizada com auxílio do software SPSS Statistics. Utilizaram-se métodos de estatística descritiva para a análise dos dados. 


\section{RESULTADOS E DISCUSSÕES}

Os temas turismo e acessibilidade têm chamado a atenção de pesquisadores nas duas últimas décadas especialmente no tocante a motivações de viagens, acessibilidade informacional, demanda de mercado e acessibilidade atitudinal (Buhalis \& Sinatra, 2019), sendo um fato a necessidade de instalações especiais para que pessoas com deficiência possam ter acesso a instalações e edifícios (Tantawy et al., 2005). Na pesquisa aplicada, 18 meios de hospedagem próximos ao Centro Histórico da cidade de Manaus (AM) participaram e tiveram como respondentes $45 \%$ colaboradores que detinham cargos de gestão e 55\% cargos operacionais. Questionados sobre o grau de conhecimento a respeito da legislação brasileira sobre os direitos da pessoa com deficiência ou mobilidade reduzida, $44,4 \%$ dos respondentes afirmaram que 0 conhecimento é superficial, havendo um desvio padrão de 0,76 , conforme se infere na Figura 1.

\section{Figura 1: Nível de conhecimento da legislação}

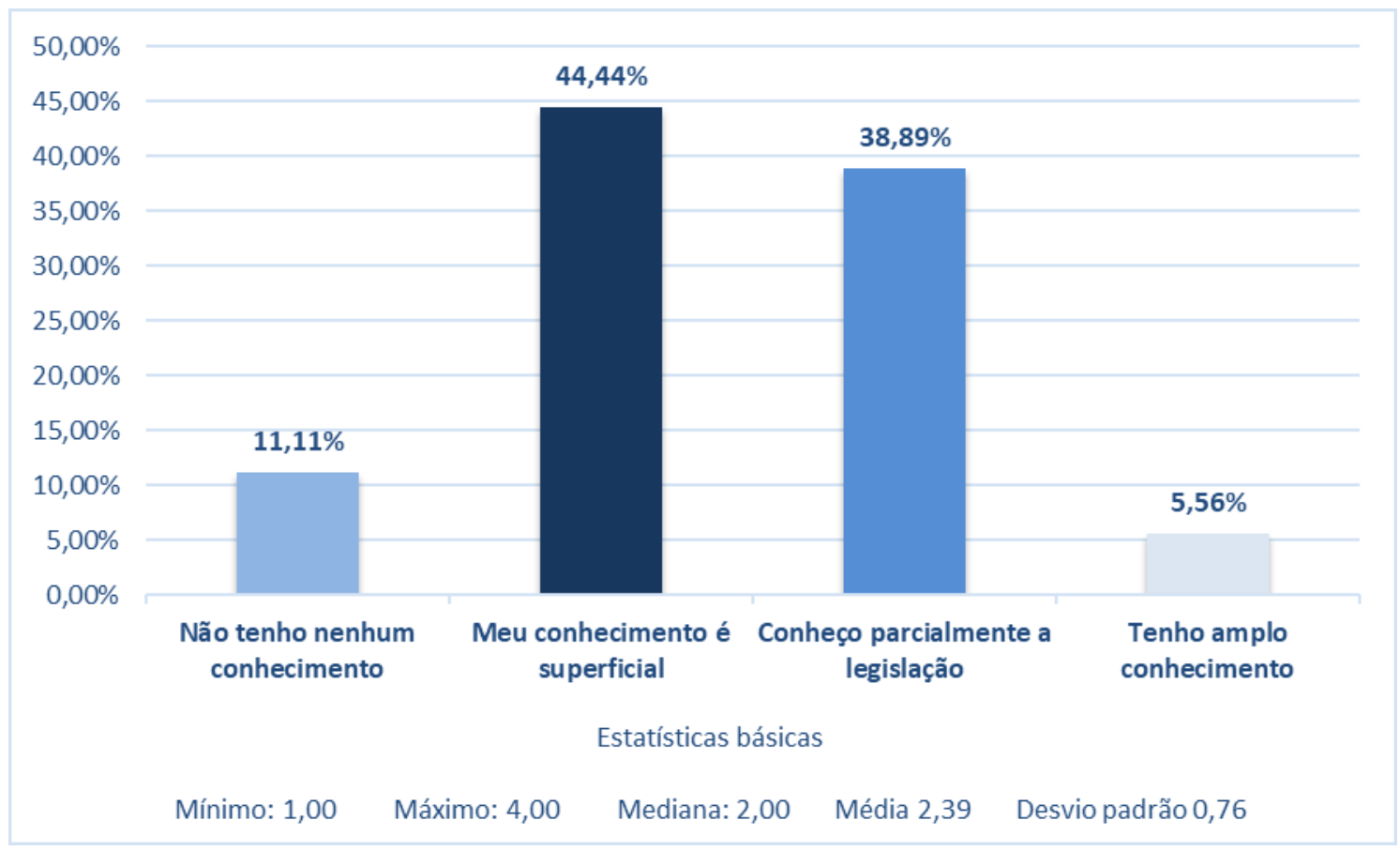

Fonte: Elaborada pelas autoras (2019).

Observou-se, ainda, que $72,22 \%$ dos meios de hospedagem nunca ofertaram um treinamento sobre os direitos da pessoa com deficiência ou mobilidade reduzida $e$ como deve ser o atendimento a este público. 
Os gestores foram questionados sobre quais competências são importantes na decisão de contratação de um funcionário para trabalhar no atendimento ao cliente. A Figura 2 demonstra a média ponderada desta escala de importância. Para o cálculo da média ponderada, foram atribuídos pesos às respostas nos seguintes critérios: 4 para imprescindível; 3 para importante, mas não essencial; 2 para importante e normalmente é considerado como um diferencial; e 1 para não é um fator que se costuma considerar.

Figura 2: Média ponderada dos elementos levados em consideração para a contratação de um funcionário para trabalhar com atendimento ao público

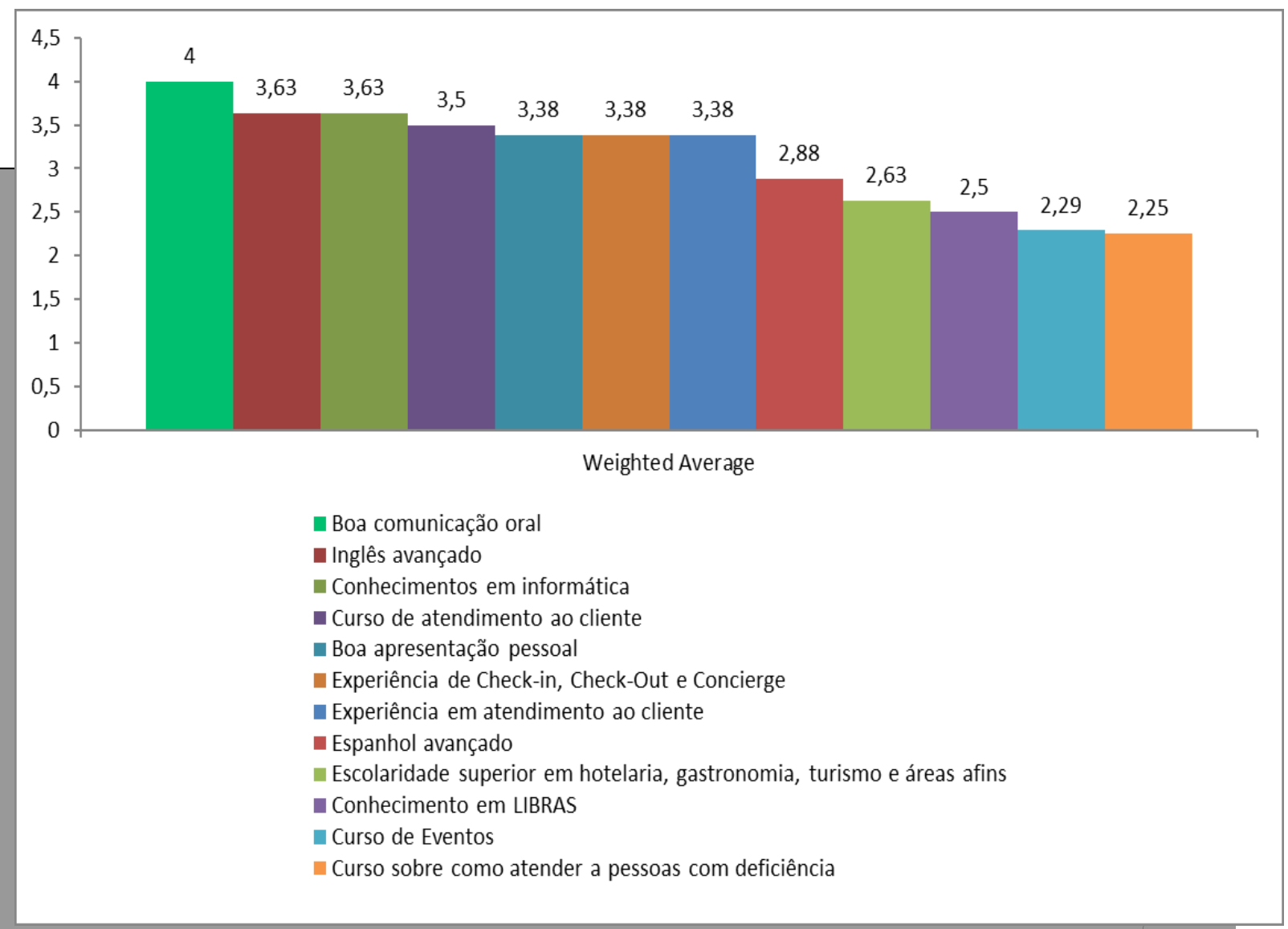

Fonte: Elaborada pelas autoras (2019).

Da análise dos dados, pode-se observar que, para 100\% dos gestores hoteleiros, a boa comunicação oral é considerada como imprescindível para a contratação, seguido de inglês avançado e informática, considerados imprescindíveis para $75 \%$ dos respondentes.

Por outro lado, para apenas $25 \%$ dos gestores é imprescindível que o candidato à vaga de trabalho tenha realizado curso sobre como atender pessoa com deficiência e conhecimentos em língua brasileira de sinais (LIBRAS).

Relativo à demanda de hóspedes com deficiência ou mobilidade reduzida, $62,50 \%$ dos gestores questionados entendem que há demanda, e informaram se preocupar em atender as necessidades deste público, enquanto que $25 \%$ dos gestores 
informaram que, em que pese entenderem haver demanda, não é um público prioritário para o empreendimento. E, por fim, para 12,50\% dos gestores, não há demanda para este segmento de clientes. Tal resultado pode ser atribuído, segundo Lyu (2017, p. 405), "à relutância social em aceitar a noção não familiar de design universal, desenvolvida para melhorar a acessibilidade geral de produtos e instalações".

No tocante à acessibilidade do meio de hospedagem, para 12,50\%, encontrase totalmente apto; para $62,50 \%$ dos gestores, o seu empreendimento está parcialmente apto; enquanto que para $25 \%$, encontra-se totalmente inapto. Vale destacar que $87,50 \%$ dos gestores entendem que a acessibilidade é um fator determinante para a escolha do local de hospedagem por uma pessoa com deficiência ou mobilidade reduzida e para $12,50 \%$ é relativamente determinante.

Entretanto, ao serem questionados sobre a disponibilização de funcionários capacitados para o atendimento da pessoa com deficiência, $75 \%$ dos gestores informaram não haver nenhum funcionário capacitado para o atendimento de pessoas com deficiência visual; auditiva e intelectual, e 50\% informaram não haver nenhum funcionário capacitado para a atendimento de pessoa com deficiência física.

Finalmente, questionados se o hotel, além do atendimento dos requisitos básicos previstos na legislação, dispõe de algum serviço diferencial, que considere inovador para o atendimento de pessoas com deficiência ou mobilidade reduzida, $100 \%$ dos gestores informaram que não, mesmo percentual encontrado nas respostas dos funcionários operacionais. Tal constatação está na contramão do que a literatura vem apresentando sobre a importância da inovação e do fornecimento de serviços personalizados como uma estratégia de sobrevivência de mercado (Meira \& Anjos, 2013, Tweneboah-Koduah et al., 2018).

Em pesquisa aplicada por Lyu (2017), demonstrou-se que a hospedagem é o atributo de maior importância para a pessoa com deficiência, que se dispõe a pagar U\$ 112,4 a mais por acomodações com alto nível de acessibilidade, o que implica a necessidade de melhor gerenciamento dos profissionais do turismo na oferta de serviços e comodidades acessíveis nas instalações de acomodação, a fim de fomentar as demandas de viagens por pessoas com deficiência ou mobilidade reduzida.

Desta feita, considerando os resultados apresentados, é importante trabalhar na sensibilização dos gestores hoteleiros sobre como os direitos da pessoa com deficiência ou mobilidade reduzida, bem como de que a utilização de processos inovadores para o atendimento das necessidades deste segmento de clientes pode contribuir significativamente para o desempenho financeiro e não financeiro do empreendimento.

\section{IMPLICAÇÕES PRÁTICAS E TEÓRICAS}

A pesquisa pode contribuir para que os gestores hoteleiros sejam sensibilizados da necessidade de investir em processos inovadores na gestão hoteleira para atender ao segmento de clientes com deficiência ou mobilidade reduzida, ante o demonstrado mercado em franca expansão, atendendo ao já indicado pela literatura da necessidade de inclusão e bem-estar no setor turístico (Michopoulou et al., 2015). 
A adoção de práticas inovadoras vem atender a uma forte demanda social, trazendo resultados importantes para a sociedade, pois se atendendo às necessidades da pessoa com deficiência, há um ganho também para os idosos, as gestantes, os obesos e as pessoas sem deficiência ou dificuldades de mobilidade.

Relativamente ao conhecimento teórico, os resultados contribuem para disseminar os elementos legais de atendimento obrigatório pelos meios de hospedagem.

\section{CONSIDERAÇÕES FINAIS}

Demonstrou-se na pesquisa realizada a inexistência de utilização de processos inovadores para o atendimento das necessidades da pessoa com deficiência ou mobilidade reduzida pelos meios de hospedagem nas proximidades do centro histórico de Manaus, bem como que a maioria dos hotéis analisados não atendem totalmente a legislação vigente no tocante ao design de acessibilidade.

Em que pese ter se demonstrado ser o turismo acessível um segmento viável e lucrativo (Prochorowicz, 2012, Rabontu, 2018, Smith et al., 2013), verificou-se que os gestores dos meios de hospedagem pesquisados entendem que a acessibilidade é um fator fundamental na escolha da acomodação pela pessoa com deficiência ou mobilidade reduzida, entretanto não tem se preocupado em atender - de forma inovadora - esta demanda.

É importante a implementação pelo poder público de sensibilização dos gestores hoteleiros sobre a importância de ofertar um ambiente acessível ao segmento de clientes com deficiência ou mobilidade reduzida, pois como se demonstrou por intermédio da literatura acadêmica, um ambiente acessível tem o condão de atrair o público com deficiência ou mobilidade reduzida, que está disposto a pagar mais por esta comodidade (Lyu, 2017). Desta feita, um meio de hospedagem altamente acessível tem um diferencial potencialmente competitivo.

Há que se ponderar, entretanto, que em virtude da dificuldade de acesso aos gestores hoteleiros, este estudo utilizou uma amostra relativamente pequena, utilizandose a metodologia de amostragem por conveniência, o que pode exigir um cuidado especial relativo à representatividade dos respondentes.

Por fim, sugerem-se estudos com uma amostra mais significativa, bem como a pesquisa da percepção do hóspede com deficiência ou mobilidade reduzida, podendo expandir-se para outras regiões geográficas.

\section{REFERÊNCIAS}

Allan, M. (2015). Accessible tourism in Jordan: Travel constrains and motivations. European Journal of Tourism Research, 10, 109-119.

Babaita, C. M. (2012). Tourism Industry in Romania and the Needs of People With Disabilities. Annals of the University of Oradea, Economic Science Series, 21 (1), 481-486. Retrieved from http://search. 
ebscohost.com/login.aspx? direct=true \&db=buh\&AN=86068778\&site=ehost-live

Biddulph, R. \& Scheyvens, R. (2018). Introducing inclusive tourism. Tourism Geographies, 20(4), 583-588. https://doi.org/10.1080/14616688.2018.1486880

BRASIL. (2008). Lei 11. 771 de 17 de setembro de 2008. Retrieved April 29, 2019, from http://www. planalto.gov.br/ccivil_03/_Ato2007-2010/2008/Lei/L1 1771.htm

BRASIL. (2015). Lei 13.146, de 6 de julho de 2015. Retrieved April 3, 2019, from http://www.planalto.gov. br/ccivil_03/_Ato2015-2018/2015/Lei/L13146.htm

BRASIL. (2018a). Decreto 9.296, de 10 de março de 2018. Retrieved April 29, 2019, from http://www. planalto.gov.br/ccivil_03/_ato2015-2018/2018/Decreto/D9296.htm

BRASIL, M. D. T. (2017). Estrutura de Pesquisa e Estudo da Demanda Turística Internacional Brasil-2017. Retrieved April 29, 2019, from http://www.dadosefatos.turismo.gov.br/images/Demanda_ Turstica_Internacional_Slides_2017.pdf

BRASIL, M. D. T. (2018b). Turismo injetou US\$163 bilhões no Brasil em 2017 - Ministério do Turismo. Retrieved March 26, 2019, from http://www.turismo.gov.br/últimas-notícias/1 1037-turismo-injetou-us\$-163bilhões-no-brasil-em-2017.html

Buhalis, D. \& Darcy, S. (2011). Accessible tourism. Concepts and Issues. Aspects of tourism. Bristol: Channel View Publications.

Buhalis, D. \& Sinarta, Y. (2019). Real-time co-creation and nowness service: lessons from tourism and hospitality. Journal of Travel \& Tourism Marketing, 36(5), 563-582. https://doi.org/10.1080/105484 08.2019.1592059

Camara, I. L. P. da \& Fonseca Filho, A. D. S. (2019). Hospitality in Rio de Janeiro as a tourist destination: perceptions of tourist exchange students of Universidade Federal Fluminense. Turismo Visão e Ação, 21 (1), 23-41. https://doi.org/10.14210/rtva.v21n1.p23-41

Card, J. A., Cole, S. T. \& Humphrey, A. H. (2006). A comparison of the Accessibility and Attitudinal Barriers Model: Travel providers and travelers with physical disabilities. Asia Pacific Journal of Tourism Research, 11 (2), 161-175. https://doi.org/10.1080/10941660600727566

Cole, S. \& Morgan, N. (2010). Tourism and Inequality : Problems and Prospects. CABI. Retrieved from https:// books.google.com.br/books/about/Tourism_and_Inequality.htmlęid=ePAckpUDP8IC\&redir_ esc $=y$

Cooper, D. R. \& Schindler, P. (2016). Métodos de pesquisa em administração (10a ed.). Porto Alegre: AMGH.

Dávid, L. \& Kiss, N. (2011). Destination development and management for disabled people. Journal of Tourism Challenges \& Trends, 4(2), 113-122.

Duarte, D. C., Borda, G.Z., Moura, D. G., \& Spexia, D. S. (2015). Accessible tourism in Brazil : an exploratory study about the public policies and the process of inclusion of persons with disabilities. Rb Tur, 9(3), 537-553. https://doi.org/http://dx.doi.org/10.7784/rbtur.v9i3.863

Hertog, P. Den, Gallouj, F. \& Segers, J. (2011). Measuring innovation in a "low-tech" service industry: The case of the Dutch hospitality industry. Service Industries Journal, 31 (9), 1429-1449. https://doi. 
org/10.1080/02642060903576084

Huh, C., \& Singh, A. J. (2007). Families Travelling with a Disabled Member: Analysing the Potential of an Emerging Niche Market Segment. Tourism and Hospitality Research, 7(4), 212-229. https://doi. org/10.1057/palgrave.thr.6050044

Jaremen, D. E. (2014). The profile of the two main social tourism segments in Poland. Ukrainian Food Journal, 3(1), 86-96.

Lyu, S. O. (2017). Which accessible travel products are people with disabilities willing to pay more? A choice experiment. Tourism Management, 59, 404-412. https://doi.org/10.1016/j.tourman.2016.09.002

Meira, J. V. de S. \& Anjos, S. J. G. dos. (2013). A Qualidade dos Serviços na Hotelaria Brasileira: A Percepção dos Gerentes Gerais e dos Gestores Setoriais (pp. 1-20). Retrieved from https://www. anptur.org.br/anais/anais/files/10/[52]x_anptur_2013.pdf

Michopoulou, E., Darcy, S., Ambrose, I. \& Buhalis, D. (2015). Accessible tourism futures: the world we dream to live in and the opportunities we hope to have. Journal of Tourism Futures, 1 (3), 179-188. https://doi.org/10.1 108/JTF-08-2015-0043

Offei, L., Acheampong, E., Appiah-Brempong, E., Okyere, P. \& Owusu, I. (2017). Accessibility of tourist sites to persons with disability: the case of Cape Coast and Elmina Castles in Ghana. Journal of Accessibility and Design for All, 7(2), 127-158. https://doi.org/10.17411/jacces.v7i2.112

Oliveira, J. P. de, Franzen, L. I. \& Varella, B. G. (2016). Acessibilidade como critério de qualidade do espaço turístico: estudo de caso da área central de Balneário Camboriú-SC. Turismo - Visão e Ação, 18(3), 660. https://doi.org/10.14210/rtva.v18n3.p660-689

Paez, P. \& Arendt, S. W. (2014). Managers' Attitudes Towards People with Disabilities in the Hospitality Industry. International Journal of Hospitality \& Tourism Administration, 15(2), 172-190. https://doi. org/10.1080/15256480.2014.901065

Prochorowicz, M. (2012). Turystyka społeczna jako forma aktywności osób niepełnosprawnych. Research Papers of the Wroclaw University of Economics, (259), 157-166. Retrieved from http:// search.ebscohost.com/login.aspx?direct=true \&db=bth\&AN=84371728\&lang=pt-br\&site=ehostlive

Rabontu, C. I. (2018). the Accessibility of Persons With Disabilities in Romanian Tourism. Revista de Turism - Studii Si Cercetari in Turism, 25, 1-6. Retrieved from http://www.revistadeturism.ro/rdt/article/ view/401

Sampieri, R. H., Collado, C. F. \& Lucio, M. D. P. B. (2015). Metodologia de pesquisa (5a ed.). Porto Alegre: Penso.

Sipe, L. J. (2018). Towards An Experience Innovation Canvas: A Framework for Measuring Innovation in the Hospitality and Tourism Industry. International Journal of Hospitality and Tourism Administration, 00(00), 1-25. https://doi.org/10.1080/15256480.2018.1547240

Smith, M., Amorim, E. \& Soares, C. (2013). O turismo acessível como vantagem competitiva: implicações na imagem do destino turístico. PASOS Revista de Turismo y Patrimonio Cultural, 11 (3), 105-121. https://doi.org/10.25145/j.pasos.2013.11.042

Tantawy, A., Kim, W. G. \& Pyo, S. (2005). Evaluation of Hotels to Accommodate Disabled Visitors 
REVISTA CIENTFIFA DO CURSO

Evaluation of Hotels to Accommodate Disabled Visitors. Jornal of Quality Assurnce in Hospitality \& Tourism, 5(April), 91-101. https://doi.org/10.1300/J162v05n01

Tweneboah-Koduah, E. Y., Anning-Dorson, T. \& Nyamekye, M. B. (2018). Impact of customization and innovation on hospitality firms' performance. Journal of Hospitality Marketing and Management, 28(04), 1-15. https://doi.org/10.1080/19368623.2019.1528917

Var, T., Yesiltas, M., Yayli, A. \& Öztürk, Y. (2011). A Study on the Travel Patterns of Physically Disabled People. Asua Pacific Journal of Tourism Research, 16(December), 37-41. https://doi.org/10.1080 $/ 10941665.2011 .610143$

Williams, V., Tarleton, B., Heslop, P., Porter, S., Sass, B., Blve, S., ... Mason-Angelow, V. (2017). Understanding disabling barriers: a fruitful partnership between Disability Studies and social practices? Disability and Society, 33(2), 157-174. https://doi.org/10.1080/09687599.2017.1401527

World Health Organization. (2012). The World Report on Disability. (SEDPCD, Ed.). São Paulo: SEDPCD. https://doi.org/10.1111/j.1468-3148.2012.00693.x

\section{Contribuição de cada autor na construção do artigo}

Marklea da Cunha Ferst: Fundamentação teórica sobre acessibilidade.

Júlia Ismar Silva de Souza: Pesquisa de campo e análise dos dados.

Helen Rita Menezes Coutinho: Metodologia e Fundamentação teórica sobre inovação. 